\title{
ДЕКОМПОЗИЦИОННЫЙ АЛГОРИТМ РЕШЕНИЯ ОДНОГО КЛАССА ЗАДАЧ МАТЕМАТИЧЕСКОГО ПРОГРАММИРОВАНИЯ
} B. ORENSTEIN. OHE MATEMAATILISE PROGRAMMEERIMISE OLESANNETEKLASSI LAHENDA-
MISEKS SOBIV DEKOMPOSITSIOONIALGORITM

B. ORENSTEIN. A DECOMPOSITION ALGORITHM FOR SOLVING A CLASS OF MATHEMATICAL PROGRAMMING PROBLEMS

В математическом программировании при кусочно-линейной аппроксимации ограничений часто возникают задачи типа: минимизировать

$$
F(x)=\sum_{j=1}^{n} f_{j}\left(x_{j}\right)
$$

при ограничениях

$$
\begin{gathered}
\sum_{j=1}^{n} d_{j} x_{j} \leqslant p, \\
a_{j} \leqslant x_{j} \leqslant b_{j}, \quad j=1,2, \ldots, n .
\end{gathered}
$$

В экономическом аспекте задачу (1)-(3) можно интерпретировать и как частный случай известной задачи распределения дефицитного реcypca.

Пусть функции $f_{j}\left(x_{j}\right)$ - строго выпуклы и дважды дифференцируемы. Без потери общности можем считать, что $d_{j} \geqslant 0, j=1,2, \ldots, n$, так как заменой определенных переменных задачу (1)-(3) всегда можно преобразовать таким образом, чтобы коэффициенты стали неотрицательными. При этом замена $x_{j} \rightarrow \widetilde{x}_{j}=-x_{j}$ для соответствующих $j$ не нарушает строгую выпуклость функции, поскольку $f_{\tilde{x}_{f}}^{\prime \prime}=f_{x_{f}}^{\prime \prime}>0$ для всех таких $j$.

В основе алгоритма решения задачи лежит правило пошагового учета ограничения (3) (этап В). При этом в качестве исходного выбирается решение ослабленной задачи $(1)-(2)$, которое вычисляется на этапе Б с использованием метода множителей Лагранжа. Этап Г учитывает случай, когда ограничение (2) не является существенным.

$\mathrm{C}$ е м а а лгори тм а. Обозначим номер шага через $i(i=0,1, \ldots)$, текущее значение правой части ограничения (2) через $p^{(i)}$ и индексы оптимизируемых компонент через $J^{(i)}$. 
Этап А. Начиная с нулевого шага $(i=0)$ положить $J(0)=$ $=\{1,2, \ldots, n\} ; p^{(0)}=p$. Проверить совместность системы неравенств (2) - (3), определяющих допустимое множество решений

$$
R=\left\{x_{j} \mid \sum_{j \in J^{(i)}} d_{j} x_{j} \leqslant p^{(i)} ; \quad a_{j} \leqslant x_{j} \leqslant b_{j}, \quad j \in J^{(i)}\right\},
$$

и, если неравенство $\sum_{j \in J^{(t)}} d_{j} a_{j} \leqslant p^{(i)}$ выполняется и $R \neq \varnothing$, перейти к этапу Б, если же $R=\varnothing$, то задача не имеет допустимых решений. Этап Б. Найти безусловный минимум $x^{*(i)}=\left(x_{1}^{*(i)}, \ldots, x_{j}^{*(i)}, \ldots, x_{n}^{*(i)}\right)$ целевой функции $(1)$ и, если $\sum_{j \in J^{(i)}} d_{j} x_{j}^{*(i)} \leqslant p^{(i)}$, перейти к этапу Г. В противном случае следует определить и $\lambda^{(i)}$ из системы

$$
\begin{aligned}
& f^{\prime}\left(x_{j}\right)-\lambda^{(i)} d_{j}=0, \quad j \in J^{(i)}, \\
& \sum_{j \in J^{(t)}} d_{j} x_{j}=p^{(i)},
\end{aligned}
$$

где $\lambda^{(i)}$ - неопределенный множитель Лагранжа.

Этап В. 1. Если $\check{J}^{(i)}=\left\{j \in J^{(i)} \mid x_{j}^{(i)}<a_{j}\right\} \neq \varnothing$, то положить $x_{j}^{\text {опт }}=a_{j}$ для $j \in \check{J}(i)$ и, приняв $i=i+1$, вернуться к этапу Б, на котором $p^{(i+1)}=p^{(i)}-\sum_{j \in \breve{J}^{(t)}} d_{j} a_{j} ; \quad J(i+1)=J(i) \breve{J}^{(i)}$.

2. Если начиная с $i=i_{0}$ окажется, что $J^{(i)}=\varnothing$, но $\hat{J(i)}=$ $=\left\{j \in J^{(i)} \mid x_{j}^{(i)}>b_{j}\right\} \neq \varnothing$, то положить $x_{j}^{\text {опт }}=b_{j}$ для $j \in \hat{J}^{(i)}$ и, приняв $i=i+1$, вернуться к этапу Б, на котором $p^{(i+1)}=p^{(i)}-\sum_{j \in \hat{J}^{(i)}} d_{j} b_{j}$; $J(i+1)=J(i) \backslash \hat{J(i)}$.

3. Если $J\left(i_{0}\right)=J\left(i_{1}\right)=\varnothing$, то оптимальное решение задачи (1)-(3) получается как упорядоченная композиция из набора $\left\{x_{j}\right\}=\left\{x_{j(r)}^{(r)}\right\}$ $r=0,1, \ldots, i_{1}$.

Этап $\Gamma$. Положить $x_{j}{ }^{*(i)}=x_{j}{ }^{(i)}, j \in J^{(i)}$, и вернуться к этапу В.

Пр и м ер. Минимизировать функцию

$$
F(x)=\sum_{j=1}^{3} f_{j}\left(x_{j}\right) ; \quad f_{j}\left(x_{j}\right)=\left(x_{j}-1\right)^{2}, \quad j \in J(0)=\{1,2,3\},
$$

при ограничениях

$$
\begin{gathered}
\sum_{j=1}^{3} x_{j} \leqslant 0,9 ; \\
0,12 \leqslant x_{1} \leqslant 0,15, \\
0,58 \leqslant x_{2} \leqslant 0,8, \\
0 \leqslant x_{3} .
\end{gathered}
$$

Этап А. Задача (6)-(8) имеет допустимое, а следовательно, и оптимальное решение, так как $\sum_{j \in J^{(0)}} d_{j} a_{j}=0,7<0,9$. Заметим, что безусловный минимум функции $(6)$ достигается в точке $x^{*}=(1 ; 1 ; 1)$, обеспечивая тем самым жесткость ограничения (7) на каждом шаге.

Этап Б. Рассмотрим систему $\left\{f^{\prime}{ }_{j}\left(x_{j}\right) \equiv 2 x_{j}=\lambda^{(0)}, \quad j \in J^{(0)}\right.$; 
$\left.x_{1}+x_{2}+x_{3}=0,9\right\}$, решение которой $x_{1}{ }^{(0)}=x_{2}{ }^{(0)}=x_{3}{ }^{(0)}=0,3 ; \lambda^{(0)}=0,6$.

Этап В. Так как $x_{2}{ }^{(0)}<0,58, \quad$ то $\check{J}^{(0)}=\{2\}, \quad x_{2}{ }^{\text {oпт }}=0,58 ; \quad p^{(1)}=$ $=0,9-0,58=0,32 ; J^{(1)}=\{1,3\}$.

На этапе Б следующего шага, решая систему $\left\{2 x_{j}=\lambda^{(1)}, j \in J^{(1)}\right.$; $\left.x_{1}+x_{3}=p^{(1)}\left(p^{(1)}=0,32\right)\right\}$, найдем $x_{1}^{(1)}=x_{3}^{(1)}=0,16 ; \lambda^{(1)}=0,32$. Здесь $\hat{J}^{(1)}=\{1\} ; x_{1}{ }^{\text {oпт }}=0,15$. Тогда $x_{3}{ }^{\text {oпт }}=0,32-0,15=0,17$ и оптимальное решение задачи $(6)-(8)$ есть $x^{\text {опт }}=(0,15 ; 0,58 ; 0,17)$.

Указанным алгоритмом решались конкретные задачи прогнозирования и планирования приема учащихся в систему высшего и среднего специального образования республики [ $\left.{ }^{1}\right]$. Для решения задач размерности 14 потребовалось в среднем четыре шага.

С ходи мость алгоритм а не более чем за $n$ шагов следует из самой его схемы, поскольку всегда найдутся натуральные числа $r$ и $s$ такие, что $\check{J}^{(r)}=\hat{J}(s)=\varnothing$, и алгоритм прекратит работу через $t=r+s$ шагов. Но $t \leqslant n$, так как $r+s \leqslant n$.

Для доказательства того, что результатом вычисления является оптимальное решение задачи (1) - (3), требуется обосновать правомерность перехода от ослабленной задачи (1)-(2) к рассматриваемой задаче (1)-(3) путем фиксации отдельных переменных на этапе В. Такой поэтапный переход основан на следующих утверждениях.

Утвер ждение 1. Если $a_{j}>x_{j}$ для $j \in \check{J}^{(i)}$, то $x_{j}^{\text {опт }}=a_{j}, \quad j \in \hat{J}^{(i)}$.

Согласно этому утверждению удается найти решение т.н. полуослабленной задачи (1)-(3)', где ограничения (3)' имеют вид

$$
a_{j} \leqslant x_{j}, \quad j=1,2, \ldots, n .
$$

Утверждение 2. Если $b_{j}<x_{j}$ для $j \in \hat{J}^{(i)}\left(i \geqslant i_{0}\right)$, то $x_{j}^{\text {опт }}=b_{j}$, $j \in \hat{J}(i)$.

Второе утверждение позволяет найти решение симметричной полуослабленной задачи (1)-(3)", где ограничения (3)" следующие:

$$
x_{j} \leqslant b_{j}, \quad j=1,2, \ldots, n .
$$

Так как учет ограничений (3)" начинается лишь после выполнения ограничений $(3)^{\prime}$, а пересчет новых значений $x_{j}$ для $j \in \hat{J}^{(i)}$ не нарушает условия (2), а следовательно, и $(3)^{\prime}$, то оптимальное решение задачи $(1)-(3)^{\prime}$, удовлетворяя ограничениям $(3)^{\prime \prime}$, является искомым.

Л И Т Р Р Т У Р А

1. Мээ л М., Изв. АН ЭССР, Общ. науки, 25, 3 (1976).

Институт экономики

Академии наук Эстонской ССР
Поступила в редакцию 31/III 1977 